\title{
Existence of Solutions for Nonconvex and Nonsmooth Vector Optimization Problems
}

\author{
Zhi-Bin Liu, ${ }^{1}$ Jong Kyu Kim, ${ }^{2}$ and Nan-Jing Huang ${ }^{3}$ \\ ${ }^{1}$ Department of Applied Mathematics, Southwest Petroleum University, Chengdu, \\ Sichuan 610500, China \\ ${ }^{2}$ Department of Mathematics, Kyungnam University, Masan, Kyungnam 631701, South Korea \\ ${ }^{3}$ Department of Mathematics, Sichuan University, Chengdu, Sichuan 610064, China
}

Correspondence should be addressed to Jong Kyu Kim, jongkyuk@kyungnam.ac.kr

Received 9 January 2008; Accepted 4 April 2008

Recommended by R. P. Gilbert

We consider the weakly efficient solution for a class of nonconvex and nonsmooth vector optimization problems in Banach spaces. We show the equivalence between the nonconvex and nonsmooth vector optimization problem and the vector variational-like inequality involving set-valued mappings. We prove some existence results concerned with the weakly efficient solution for the nonconvex and nonsmooth vector optimization problems by using the equivalence and Fan-KKM theorem under some suitable conditions.

Copyright ( $) 2008$ Zhi-Bin Liu et al. This is an open access article distributed under the Creative Commons Attribution License, which permits unrestricted use, distribution, and reproduction in any medium, provided the original work is properly cited.

\section{Introduction}

The concept of vector variational inequality was first introduced by Giannessi [1] in 1980. Since then, existence theorems for solution of general versions of the vector variational inequality have been studied by many authors (see, e.g., [2-9] and the references therein). Recently, vector variational inequalities and their generalizations have been used as a tool to solve vector optimization problems (see [7, 10-14]). Chen and Craven [11] obtained a sufficient condition for the existence of weakly efficient solutions for differentiable vector optimization problems involving differentiable convex functions by using vector variational inequalities for vector valued functions. Kazmi [12] proved a sufficient condition for the existence of weakly efficient solutions for vector optimization problems involving differentiable preinvex functions by using vector variational-like inequalities. For the nonsmooth case, Lee et al. [7] established the existence of the weakly efficient solution for nondifferentiable vector optimization problems by using vector variational-like inequalities for set-valued mappings. Similar results can be found in [10]. It is worth mentioning that Lee et al. [7] and Ansari and Yao [10] obtained their 
existence results under the assumption that $R_{+}^{m} \subset C(x)$ for all $x \in R^{n}$, where $C(x)$ is a convex cone in $R^{m}$. However, this condition is restrict and it does not hold in general.

In this paper, we consider the weakly efficient solution for a class of nonconvex and nonsmooth vector optimization problems in Banach spaces. We show the equivalence between the nonconvex and nonsmooth vector optimization problem and the vector variational-like inequality involving set-valued mappings. We prove some existence results concerned with the weakly efficient solution for the nonconvex and nonsmooth vector optimization problems by using the equivalence and Fan-KKM theorem without the restrict condition $R_{+}^{m} \subset C(x)$ for all $x \in R^{n}$. Our results generalize and improve the results obtained by Lee et al. [7] and Ansari and Yao [10].

\section{Preliminaries}

Let $X$ be a real Banach space endowed with a norm $\|\cdot\|$ and $X^{*}$ its dual space, we denote by $\langle\cdot, \cdot\rangle$ the dual pair between $X$ and $X^{*}$. Let $R^{m}$ be the $m$-dimensional Euclidean space, let $S \subset X$ be a nonempty subset, and let $K \subset R^{m}$ be a nonempty closed convex cone with int $K \neq \varnothing$, where int denotes interior.

Definition 2.1. A real valued function $h: X \rightarrow R$ is said to be locally Lipschitz at a point $x \in X$ if there exists a number $L>0$ such that

$$
|h(y)-h(z)| \leq L\|y-z\|
$$

for all $y, z$ in a neighborhood of $x . h$ is said to be locally Lipschitz on $X$ if it is locally Lipschitz at each point of $X$.

Definition 2.2. Let $h: X \rightarrow R$ be a locally Lipschitz function. Clarke [15] generalized directional derivative of $h$ at $x \in X$ in the direction $v$, denoted by $h^{\circ}(x ; v)$, is defined by

$$
h^{\circ}(x ; v)=\limsup _{y \rightarrow x, t \downarrow 0} \frac{h(y+t v)-h(y)}{t} .
$$

Clarke [15] generalized gradient of $h$ at $x \in X$, denoted by $\partial h(x)$, is defined by

$$
\partial h(x)=\left\{\xi \in X^{*}: h^{\circ}(x ; v) \geq\langle\xi, d\rangle \forall v \in X\right\} .
$$

Let $f: X \rightarrow R^{m}$ be a vector valued function given by $f=\left(f_{1}, f_{2}, \ldots, f_{m}\right)$, where each $f_{i}, i=$ $1,2, \ldots, m$, is a real valued function defined on $X$. Then $f$ is said to be locally Lipschitz on $X$ if each $f_{i}$ is locally Lipschitz on $X$.

The generalized directional derivative of a locally Lipschitz function $f: X \rightarrow R^{m}$ at $x \in X$ in the direction $v$ is given by

$$
f^{\circ}(x ; v)=\left(f_{1}^{\circ}(x ; v), f_{2}^{\circ}(x ; v), \ldots, f_{m}^{\circ}(x ; v)\right) .
$$

The generalized gradient of $h$ at $x$ is the set

$$
\partial f(x)=\partial f_{1}(x) \times \partial f_{2}(x) \times \cdots \times \partial f_{m}(x),
$$

where $\partial f_{i}(x)$ is the generalized gradient of $f_{i}$ at $x$ for $i=1,2, \ldots, m$. and

Every element $A=\left(\xi_{1}, \xi_{2}, \ldots, \xi_{m}\right) \in \partial f(x)$ is a continuous linear operator from $X$ to $R^{m}$

$$
A y=\left(\left\langle\xi_{1}, y\right\rangle,\left\langle\xi_{2}, y\right\rangle, \ldots,\left\langle\xi_{m}, y\right\rangle\right) \in R^{m}, \quad \forall y \in X
$$


Definition 2.3. Let $f: X \rightarrow R^{m}$ be a locally Lipschitz function.

(i) $f$ is said to be $K$-invex with respect to $\eta$ at $u \in X$, if there exists $\eta: X \times X \rightarrow X$ such that for all $x \in X$ and $A \in \partial f(u)$,

$$
f(x)-f(u)-\langle A, \eta(x, u)\rangle \in K
$$

(ii) $f$ is said to be $K$-pseudoinvex with respect to $\eta$ at $u \in X$ if there exists $\eta: X \times X \rightarrow X$ such that for all $x \in X$ and $A \in \partial f(u)$,

$$
f(x)-f(u) \in-\operatorname{int} K \Longrightarrow\langle A, \eta(x, u)\rangle \in-\operatorname{int} K \text {. }
$$

In this paper, we consider the following nonsmooth vector optimization problem:

$$
\begin{gathered}
\text { K-minimize } f(x), \\
\text { subject to } x \in S,
\end{gathered}
$$

where $f=\left(f_{1}, f_{2}, \ldots, f_{m}\right), f_{i}: X \rightarrow R, i=1,2, \ldots, m$, are locally Lipschitz functions.

Definition 2.4. A point $x_{0} \in S$ is said to be a weakly efficient solution of $f$ if there exists no $y \in S$ such that

$$
f(y)-f(x) \in-\operatorname{int} K
$$

In order to prove our main results, we need the following definition and lemmas.

Definition 2.5 (see [16]). A multivalued mapping $G: X \rightarrow 2^{X}$ is called KKM-mapping if for any finite subset $\left\{x_{1}, x_{2}, \ldots, x_{n}\right\}$ of $X, \operatorname{co}\left\{x_{1}, x_{2}, \ldots, x_{n}\right\}$ is contained in $\bigcup_{i=1}^{n} G\left(x_{i}\right)$, where $\operatorname{co} A$ denotes the convex hull of the set $A$.

Lemma 2.6 (see [16]). Let $M$ be a nonempty subset of a Hausdorff topological vector space X. Let $G: M \rightarrow 2^{X}$ be a KKM-mapping such that $G(x)$ is closed for any $x \in M$ and is compact for at least one $x \in M$. Then $\bigcap_{y \in M} G(y) \neq \varnothing$.

Lemma 2.7 (see [2]). Let $K$ be a convex cone of topological vector space $X$. If $y-x \in K$ and $x \notin-i n t K$, then $y \notin-\operatorname{int} K$ for any $x, y \in X$.

\section{Main results}

In order to obtain our main results, we introduce the following vector variational-like inequality problem, which consists in finding $x_{0} \in S$ such that for all $A \in \partial f\left(x_{0}\right)$,

$$
\left\langle A, \eta\left(y, x_{0}\right)\right\rangle \notin-\operatorname{int} K, \quad \forall y \in S
$$

First, we establish the following relations between (VOP) and (VVIP). 
Lemma 3.1. Let $f: X \rightarrow R^{m}$ be a locally Lipschitz function and $\eta: S \times S \rightarrow X$. Then the following arguments hold.

(i) Suppose that $f$ is $K$-invex with respect to $\eta$. If $x_{0}$ is a solution of (VVIP), then $x_{0}$ is a weakly efficient solution of (VOP).

(ii) Suppose that $f$ is $K$-pseudoinvex with respect to $\eta$. If $x_{0}$ is a solution of (VVIP), then $x_{0}$ is a weakly efficient solution of (VOP).

(iii) Suppose that $f$ is $-K$-invex with respect to $\eta$. If $x_{0}$ is a weakly efficient solution of (VOP), then $x_{0}$ is a solution of (VVIP).

Proof. (i) Let $x_{0}$ be a solution of (VVIP). Then

$$
\left\langle A, \eta\left(y, x_{0}\right)\right\rangle \notin-\operatorname{int} K, \quad \forall A \in \partial f\left(x_{0}\right), y \in S .
$$

By the $K$-invexity of $f$ with respect to $\eta$, we get

$$
f(y)-f\left(x_{0}\right)-\left\langle A, \eta\left(y, x_{0}\right)\right\rangle \in K, \quad \forall A \in \partial f\left(x_{0}\right), y \in S
$$

From (3.1), (3.2) and Lemma 2.7, we obtain

$$
f(y)-f\left(x_{0}\right) \notin-\operatorname{int} K, \quad \forall y \in S
$$

Therefore, $x_{0}$ is a weakly efficient solution of (VOP).

(ii) Let $x_{0}$ be a solution of (VVIP). Suppose that $x_{0}$ is not a weakly efficient solution of (VOP). Then, there exists $y \in S$ such that

$$
f(y)-f\left(x_{0}\right) \in-\operatorname{int} K
$$

Since $f$ is $K$-pseudoinvex with respect to $\eta$, then

$$
\left\langle A, \eta\left(y, x_{0}\right)\right\rangle \in-\operatorname{int} K, \quad \forall A \in \partial f\left(x_{0}\right),
$$

which contradicts the fact that $x_{0}$ is a solution of (VVIP).

(iii) Assume that $x_{0}$ is a weakly efficient solution of (VOP). Then,

$$
f(y)-f\left(x_{0}\right) \notin-\operatorname{int} K, \quad \forall y \in S
$$

Since $f$ is $-K$-invex with respect to $\eta$, then

$$
f(y)-f\left(x_{0}\right)-\left\langle A, \eta\left(y, x_{0}\right)\right\rangle \in-K, \quad \forall A \in \partial f\left(x_{0}\right), y \in S .
$$

It follows from Lemma 2.7 that

$$
\left\langle A, \eta\left(y, x_{0}\right)\right\rangle \notin-\operatorname{int} K, \quad \forall A \in \partial f\left(x_{0}\right), y \in S
$$

Therefore, $x_{0}$ is a solution of (VVIP). 
Now we establish the following existence theorem.

Theorem 3.2. Let $S \subset X$ be a nonempty convex set and $\eta: S \times S \rightarrow X$. Let $f: X \rightarrow R^{m}$ be a locally Lipschitz K-pseudoinvex function. Assume that the following conditions hold

(i) $\eta(x, x)=0$ for any $x \in S, \eta(y, x)$ is affine with respect to $y$ and continuous with respect to $x$;

(ii) there exist a compact subset $D$ of $S$ and $y_{0} \in D$ such that

$$
\left\langle A, \eta\left(y_{0}, x\right)\right\rangle \in-\operatorname{int} K, \quad \forall x \in S \backslash D, A \in \partial f(x) .
$$

Then (VOP) has a weakly efficient solution.

Proof. By Lemma 3.1(ii), it suffices to prove that (VVIP) has a solution. Define $G: S \rightarrow 2^{S}$ by

$$
G(y)=\{x \in S:\langle A, \eta(y, x)\rangle \notin-\operatorname{int} K, \forall A \in \partial f(x)\}, \quad \forall y \in S .
$$

First we show that $G$ is a KKM-mapping. By condition (i), we get $y \in G(y)$. Hence, $G(y) \neq \varnothing$ for all $y \in S$. Suppose that there exists a finite subset $\left\{x_{1}, x_{2}, \ldots, x_{m}\right\} \subseteq S$ and that $\alpha_{i} \geq 0, i=1,2, \ldots, m$, with $\sum_{i=1}^{m} \alpha_{i}=1$ such that $x=\sum_{i=1}^{m} \alpha_{i} x_{i} \notin \bigcup_{i=1}^{m} G\left(x_{i}\right)$. Then, $x \notin G\left(x_{i}\right)$ for all $i=1,2, \ldots, m$. It follows that there exists $A \in \partial f(x)$ such that

$$
\left\langle A, \eta\left(x_{i}, x\right)\right\rangle \in-\text { int } K, \quad i=1,2, \ldots, m \text {. }
$$

Since $K$ is a convex cone and $\eta$ is affine with respect to the first argument,

$$
\langle A, \eta(x, x)\rangle \in-\text { int } K \text {. }
$$

which gives $0 \in-\operatorname{int} K$. This is a contradiction since $0 \notin-\operatorname{int} K$. Therefore, $G$ is a KKMmapping.

Next, we show that $G(y)$ is a closed set for any $y \in S$. In fact, let $\left\{x_{n}\right\}$ be a sequence of $G(y)$ which converges to some $x_{0} \in S$. Then for all $A_{n} \in \partial f\left(x_{n}\right)$, we have

$$
\left\langle A_{n}, \eta\left(y, x_{n}\right)\right\rangle \notin-\operatorname{int} K \text {. }
$$

Since $f$ is locally Lipschitz, then there exists a neighborhood $N\left(x_{0}\right)$ of $x_{0}$ and $L>0$ such that for any $x, y \in N\left(x_{0}\right)$,

$$
|f(x)-f(y)| \leq L\|x-y\| .
$$

It follows that for any $x \in N\left(x_{0}\right)$ and any $A \in \partial f(x),\|A\| \leq L$. Without loss of generality, we may assume that $A_{n}$ converges to $A_{0}$. Since the set-valued mapping $x \mapsto \partial f(x)$ is closed (see [15, page 29]) and $A_{n} \in \partial f\left(x_{n}\right), A_{0} \in \partial f\left(x_{0}\right)$. By the continuity of $\eta(y, x)$ with respect to the second argument, we have

$$
\left\langle A_{n}, \eta\left(y, x_{n}\right)\right\rangle \longrightarrow\left\langle A_{0}, \eta\left(y, x_{0}\right)\right\rangle
$$

Since $R^{m} \backslash$-int $K$ is closed, one has

$$
\left\langle A_{0}, \eta\left(y, x_{0}\right)\right\rangle \notin-\operatorname{int} K .
$$

Hence, $G(y)$ is a closed set for any $y \in S$. 
By condition (ii), we have $G\left(y_{0}\right) \subset D$. As $G\left(y_{0}\right)$ is closed and $D$ is compact, $G\left(y_{0}\right)$ is compact. Therefore, by Lemma 2.6, we have that there exists $x^{*} \in S$ such that

$$
x^{*} \in \bigcap_{y \in S} G(y),
$$

or equivalently,

$$
\left\langle A, \eta\left(y, x^{*}\right)\right\rangle \notin-\operatorname{int} K, \quad \forall A \in \partial f\left(x^{*}\right), y \in S
$$

That is, $x^{*}$ is a solution of (VVIP). This completes the proof.

Corollary 3.3. Let $S \subset X$ be a nonempty convex set and $\eta: S \times S \rightarrow X$. Let $f: X \rightarrow R^{m}$ be a locally Lipschitz K-invex function. Assume that the following conditions hold:

(i) $\eta(x, x)=0$ for any $x \in S, \eta(y, x)$ is affine with respect to $y$ and continuous with respect to $x$;

(ii) there exist a compact subset $D$ of $S$ and $y_{0} \in D$ such that

$$
\left\langle A, \eta\left(y_{0}, x\right)\right\rangle \in-\operatorname{int} K, \quad \forall x \in S \backslash D, A \in \partial f(x) .
$$

Then (VOP) has a weakly efficient solution.

Proof. Since a $K$-invex function is $K$-pseudoinvex, by Theorem 3.2, we obtain the result.

\section{Acknowledgments}

This work was supported by the National Natural Science Foundation of China (10671135), the Specialized Research Fund for the Doctoral Program of Higher Education (20060610005) and the Open Fund (PLN0703) of State Key Laboratory of Oil and Gas Reservoir Geology and Exploitation (Southwest Petroleum University). And J. K. Kim was supported by the Korea Research Fundation Grant funded by the Korean Goverment (MOEHRD, Basic Research Promotion Fund)(KRF-2006-311-C00201.)

\section{References}

[1] F. Giannessi, "Theorems of alternative, quadratic programs and complementarity problems," in Variational Inequalities and Complementarity Problems, R. W. Cottle, F. Giannessi, and J. L. Lions, Eds., pp. 151-186, John Wiley \& Sons, Chichester, UK, 1980.

[2] G. Y. Chen, "Existence of solutions for a vector variational inequality: an extension of the HartmannStampacchia theorem," Journal of Optimization Theory and Applications, vol. 74, no. 3, pp. 445-456, 1992.

[3] F. Giannessi, Ed., Vector Variational Inequalities and Vector Equilibria. Mathematical Theories, vol. 38 of Nonconvex Optimization and Its Applications, Kluwer Academic Publishers, Dordrecht, The Netherlands, 2000.

[4] N.-J. Huang and Y.-P. Fang, "On vector variational inequalities in reflexive Banach spaces," Journal of Global Optimization, vol. 32, no. 4, pp. 495-505, 2005.

[5] N.-J. Huang and J. Li, "On vector implicit variational inequalities and complementarity problems," Journal of Global Optimization, vol. 34, no. 3, pp. 399-408, 2006.

[6] I. V. Konnov and J. C. Yao, "On the generalized vector variational inequality problem," Journal of Mathematical Analysis and Applications, vol. 206, no. 1, pp. 42-58, 1997. 
[7] G. M. Lee, D. S. Kim, and H. Kuk, "Existence of solutions for vector optimization problems," Journal of Mathematical Analysis and Applications, vol. 220, no. 1, pp. 90-98, 1998.

[8] G. M. Lee, B. S. Lee, and S.-S. Chang, "On vector quasivariational inequalities," Journal of Mathematical Analysis and Applications, vol. 203, no. 3, pp. 626-638, 1996.

[9] S. J. Yu and J. C. Yao, "On vector variational inequalities," Journal of Optimization Theory and Applications, vol. 89, no. 3, pp. 749-769, 1996.

[10] Q. H. Ansari and J. C. Yao, "On nondifferentiable and nonconvex vector optimization problems," Journal of Optimization Theory and Applications, vol. 106, no. 3, pp. 475-488, 2000.

[11] G. Y. Chen and B. D. Craven, "Existence and continuity of solutions for vector optimization," Journal of Optimization Theory and Applications, vol. 81, no. 3, pp. 459-468, 1994.

[12] K. R. Kazmi, "Some remarks on vector optimization problems," Journal of Optimization Theory and Applications, vol. 96, no. 1, pp. 133-138, 1998.

[13] G. M. Lee, D. S. Kim, B. S. Lee, and N. D. Yen, "Vector variational inequality as a tool for studying vector optimization problems," Nonlinear Analysis: Theory, Methods \& Applications, vol. 34, no. 5, pp. 745-765, 1998.

[14] X. Q. Yang, "Generalized convex functions and vector variational inequalities," Journal of Optimization Theory and Applications, vol. 79, no. 3, pp. 563-580, 1993.

[15] F. H. Clarke, Optimization and Nonsmooth Analysis, Canadian Mathematical Society Series of Monographs and Advanced Texts, John Wiley \& Sons, New York, NY, USA, 1983.

[16] K. Fan, "A generalization of Tychonoff's fixed point theorem," Mathematische Annalen, vol. 142, no. 3 , pp. 305-310, 1961. 